\title{
Tuberculous Peritonsillar Abscess: A Case Report in Malaysia
}

\author{
Farid Razali', Ihab E. Ali' ${ }^{2}$, K. Y. Loh ${ }^{3}$ \\ ${ }^{1}$ ENT Department, Hospital Sungai Buloh, Malaysia \\ ${ }^{2}$ ENT Department, Taylor's University, Subang Jaya, Selangor, Malaysia \\ ${ }^{3}$ Family Medicine Department, Taylor's University, Subang Jaya, Selangor, Malaysia \\ Email:ihab.ali@taylors.edu.my
}

How to cite this paper: Razali, F., Ali, I.E. and Loh, K.Y. (2019) Tuberculous Peritonsillar Abscess: A Case Report in Malaysia. International Journal of Otolaryngology and Head \& Neck Surgery, 8, 166-170. https://doi.org/10.4236/ijohns.2019.85018

Received: July 11, 2019

Accepted: August 19, 2019

Published: August 22, 2019

Copyright $\odot 2019$ by author(s) and Scientific Research Publishing Inc. This work is licensed under the Creative Commons Attribution International License (CC BY 4.0).

http://creativecommons.org/licenses/by/4.0/

\begin{abstract}
Tonsillitis is one of the most common presentations to the primary care clinic. Majority of the tonsillitis is viral etiology and occasionally is caused by bacteria such as streptococcal pharyngo-tonsillitis. However, if tonsillitis lasts longer than a week and associated with cervical lymph nodes, other differential diagnoses should be considered. This case report illustrated a patient who presented with fever, sore throat and odynophagia which lasted for one week then developed trismus. Examination revealed a left tonsillar hypertrophy with soft palate swelling and pus aspirated from the tonsil revealed positive smear to Acid-fast Bacilli and he responded to anti-tuberculosis treatment.
\end{abstract}

\section{Keywords}

Tonsillitis, Abscess, Tuberculosis

\section{Introduction}

Acute pharyngeal tonsillitis is a common presentation seen in primary care worldwide. In Malaysia, it is one of the most common complaints that patients visit their family doctor. Majority of acute pharyngeal tonsillitis seen is caused by viral infection and most of the patients require only symptomatic treatment. Occasionally more severe cases are caused by Streptococcal bacteria. However, if a patient is diagnosed with acute pharyngeal tonsillitis which is not responding to symptomatic treatment, he should be examined further. There may be other rare causes, or the patient may have developed complications from the acute phase. The following case illustrated a usual presentation of sore throat and fever which lasted for one week associated with odynophagia. Follow-up review of this patient confirmed tuberculosis as the cause of his persistent symptoms. 


\section{History}

A 33-year-old Malay man was referred from Emergency Department to The Department of Otorhinolaryngology with a history of persistent sore throat of one-week duration associated with odynophagia. He also had low grade fever for the past one week when the sore throat started. There was no history of rhinitis, cough or hoarseness of voice. He had no significant past history of medical illness and no known illness in his family. There was no history of contact with any people who diagnosed with any infectious disease. There was also no known event of immune deficiency or any close contact with tuberculosis patient.

On examination, he was pink and has medium size body and no significant muscle wasting.

\begin{tabular}{cc}
\hline Blood pressure & $150 / 90 \mathrm{Mm} \mathrm{Hg}$ \\
Pulse rate & 90 beats per minute and regular \\
Temperature & 38 degrees Centigrade \\
\hline
\end{tabular}

There was some palpable shorty cervical lymph nodes present.

Examination of the oral cavity appeared normal, with trismus. However, examination of his oropharyngeal region revealed enlarged tonsils grade 4 on the left side and grade 3 on the right side. The left peritonsillar region was red, inflamed and it was boggy with pulling of the soft palate. Uvula was deviated to the right side. A working diagnosis of tonsillar abscess was established, the abscess was completely drained (Figure 1), and further investigations were then carried out to determine the underlying cause. Examination of the cardiovascular, respiratory and abdomen were unremarkable. Aspiration of the pus from the abscess site was sent for Culture \& Sensitivity test and AFB test.

\begin{tabular}{cc}
\hline Hemoglobin & $14 \mathrm{gm} \%$ \\
Total white cell counts & $15 \times 10^{9} / \mathrm{L}$ \\
\hline
\end{tabular}

His plain chest radiograph showed mild haziness at the left hilar region, which was highly suspicious of pulmonary tuberculosis (Figure 2). A sputum direct smear was found to be positive for Acid Fast Bacilli with Ziehl-Neelsenstained, also the aspirate for AFB came back as positively for Acid Fast Bacilli. A final diagnosis of tuberculoustonsillar abscess was established, and he was started with anti-tuberculosis treatment.

\section{Progress}

$\mathrm{CT}$ of the neck region revealed peritonsillar abscess but no extension to the parapharyngeal and retropharyngeal spaces. The Nasopharynx and laryngopharynx were normal, and the paranasal sinuses were normal too (Figure 3). The patient was followed up more than one year, he started to respond once he started the anti-tuberculosis treatment, he showed some improvement after 3 


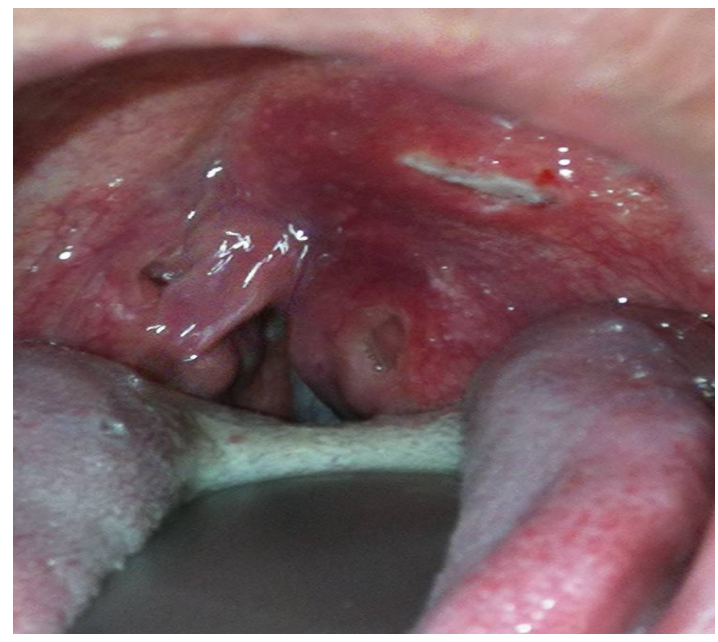

Figure 1. The Peritonsillar abscess after drainage.

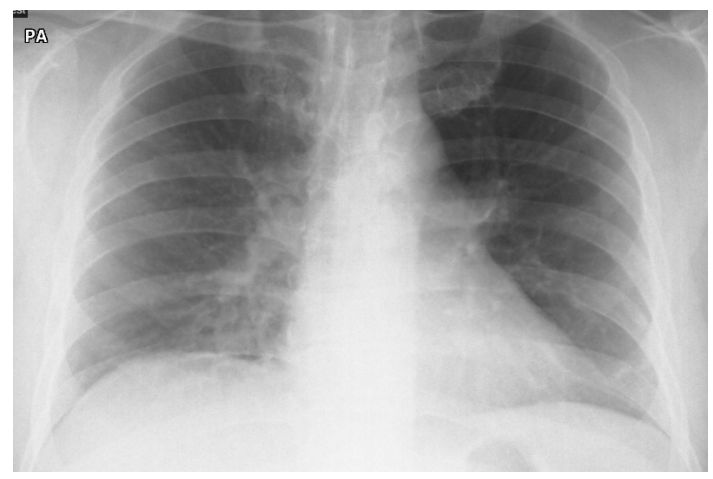

Figure 2. Left perihilar haziness.

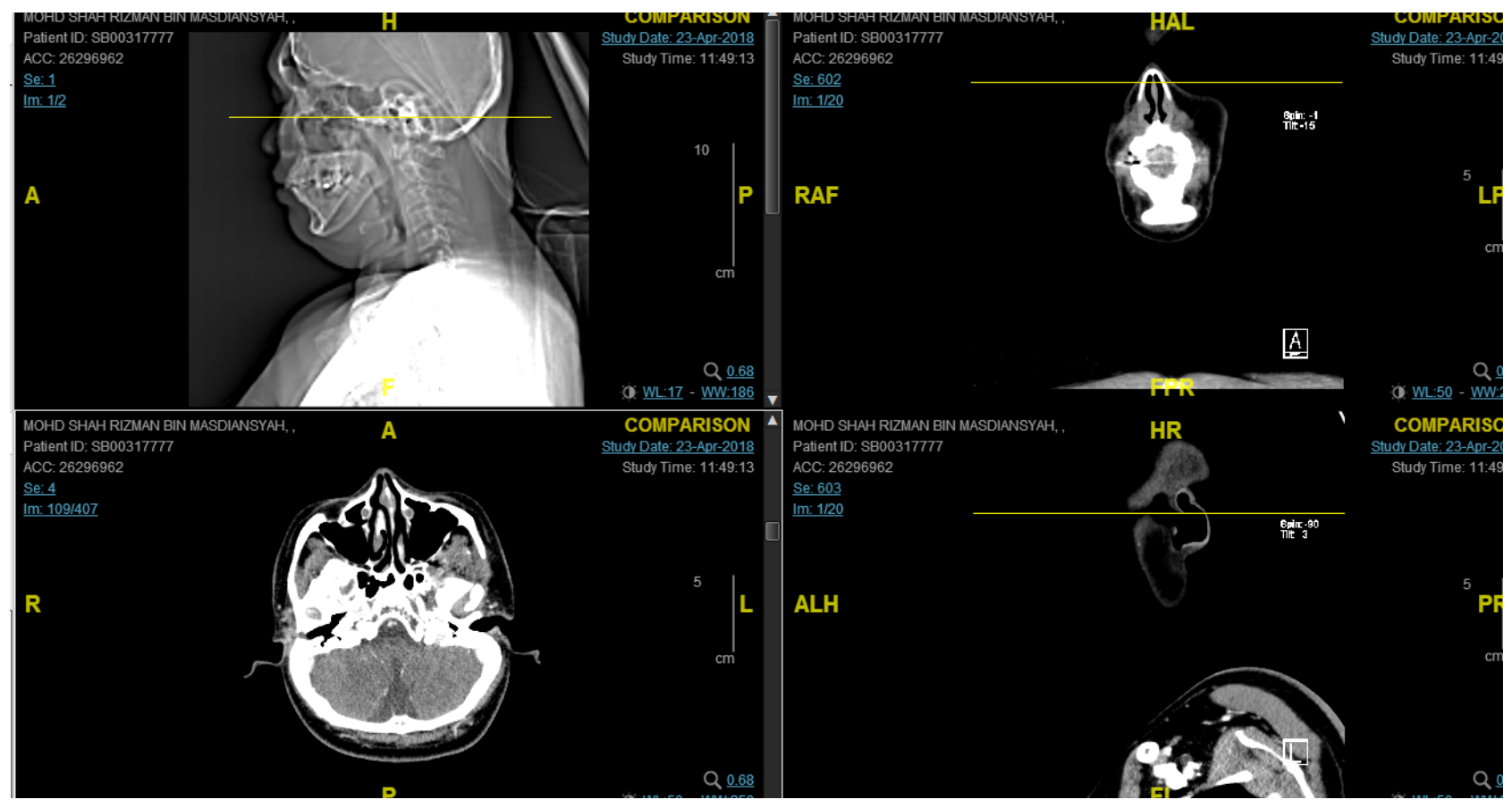

Figure 3. Left peritonsillar abscess. The parapharyngeal and retropharyngeal spaces are clear. 
month of taking the treatment, a lot of improvement after 6 months of treatment and complete resolution after 9 months of getting the anti-tuberculosis treatment. Currently he is well and the clinical symptoms had since subsided. He will continue to be followed up for further 1 year to ensure there is no complication. His close family members underwent tuberculosis screening but none of them has positive result and sent for treatment.

\section{Discussion}

Peritonsillar abscess also known as quinsy is a complication following acute bacterial tonsillitis. Group A beta hemolytic streptococcus (Streptococcus pyogenes) is one of the most common causes in children and young adults, other etiological agents include Staphylococcus aureus, Bacteroides, Hemophilussp and other anaerobes [1]. Majority of the affected population are young adults and there is no difference in the prevalence in male and female [2].

Primary tuberculosis of the tonsils is rare, it is usually a sequelae following pulmonary tuberculosis [3]. There was a published report that up to $6.5 \%$ of removed tonsils were found to be infected by tubercle [4]. In Malaysia, prevalence of pulmonary tuberculosis is rising over the past ten years particularly associated with Human Immunodeficiency Virus infection [5]. However, there is no published data on the prevalence of peritonsillar abscess caused by tuberculosis in Malaysia. Other immunocompromised conditions that predispose a person to tuberculoustonsillar abscess are diabetes mellitus and chronic alcoholics.

Common symptoms that bring a patient to seek treatment for peritonsillar abscess are persistent pain, difficulty in swallowing, pain on swallowing or persistent systemic symptoms such as fever and lethargy. Without proper treatment peritonsillar can lead to an otolaryngologic emergency [2]. In this patient presented here, the similar symptoms occurred. As with other patients, most of these symptoms were treated as usual viral pharyngeal tonsillitis. One of the most crucial points which we would like to highlight here is that persistent systemic symptom such as fever and painful swallowing that is not resolved after one week should alert the treating doctor to think of alternative diagnosis or to investigate further for underlying causes. High index of suspicion should be kept in mind when evaluating a patient presenting with sore throat but non resolving symptoms following one week of conservative management.

The most important management for tuberculousperitonsillar abscess should be combination of drainage of abscess together with anti-tuberculosis treatment. This patient responded well to the management and recovered fully. Without proper management, tuberculoustonsillitis can cause serious complications such as extending abscess that causes respiratory distress and systemic complications of military tuberculosis.

This case illustrated the importance of recognizing tuberculosis as a cause of tonsillar abscess in a patient coming from tuberculosis endemic area. Persistent symptoms despite conservative treatment of usual pharyngeal tonsillitis in pri- 
mary care clinic should be referred to otorhinolaryngology clinic for further evaluation to look for alternative diagnosis. This patient is also a young man who has no known medical illness and no history of contact with any patient diagnosed with tuberculosis. Pulmonary tuberculosis in Malaysia is common, therefore it should be considered as one of the etiological agent in persistent severe tonsillitis with abscess formation in this country.

\section{Conflicts of Interest}

The authors declare no conflicts of interest regarding the publication of this paper.

\section{References}

[1] Brook, I., Frazier, E.H. and Thompson, D.H. (1991) Aerobic and Anaerobic Microbiology of Peritonsillar Abscess. Laryngoscope, 101, 289-292. https://doi.org/10.1288/00005537-199103000-00012

[2] Afolabi, O.A., et al. (2014) Peritonsillar Abscess in Northern Nigeria: A 7 Years Review. Malaysian Journal of Medical Sciences, 21, 14-18.

[3] Panduranga, M.K., Shenoy, V.S., Nirupama, M., Prasad, V. and Majeed, N.Z. (2015) Tuberculosis of Waldeyer's Ring with an Atypical Presentation as Chronic Adeno-Tonsilitis. Journal of Clinical \& Diagnostic Research, 9, MD01-MD02.

[4] Shalini, J., Chauhan, A.N., Hazari, R.S., Maurya, A.K. and Biswas, B. (2010) An Unusual Cause of Recurrent Tonsillitis. BMJ Case Reports. https://doi.org/10.1136/bcr.12.2009.2561

[5] Rohan, S. and Indra Devi, S. (2015) Clinical Manifestation and Risk Factors of Tuberculosis Infection in Malaysia: Case Study of a Community Clinic. Global Journal of Health Science, 7, 110-120. https://doi.org/10.5539/gjhs.v7n4p110 